\title{
Challenges to Speech Perception Impair Phonological Short-Term Memory
}

Harriet J. Smith, Matthew H. Davis MRC Cognition and Brain Sciences Unit, 15 Chaucer Road, Cambridge CB2 7EF

\section{BACKGROUND}

Poor performance on phonological tasks is a key feature of neurodevelopmental language disorders (Snowling, 1998;

Ramus et al., 2013).

Perceptual deficit accounts link phonological dysfunction to deficits in speech-sound discrimination at lower levels of the speech processing hierarchy. A cognitively realistic pathway from speech perception to phonological performance has not been sufficiently established. We assessed this relationship in typical adults by experimentally disrupting speech-sound discrimination in a phonological short-term memory (pSTM) task.

\section{Causal Model}

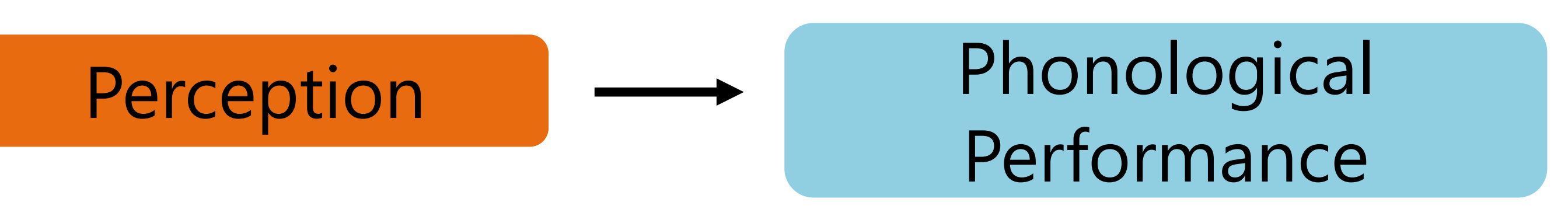

\section{Consequence Model}

Phonological Performance $\longrightarrow$ Perception

\section{Correlation Model}

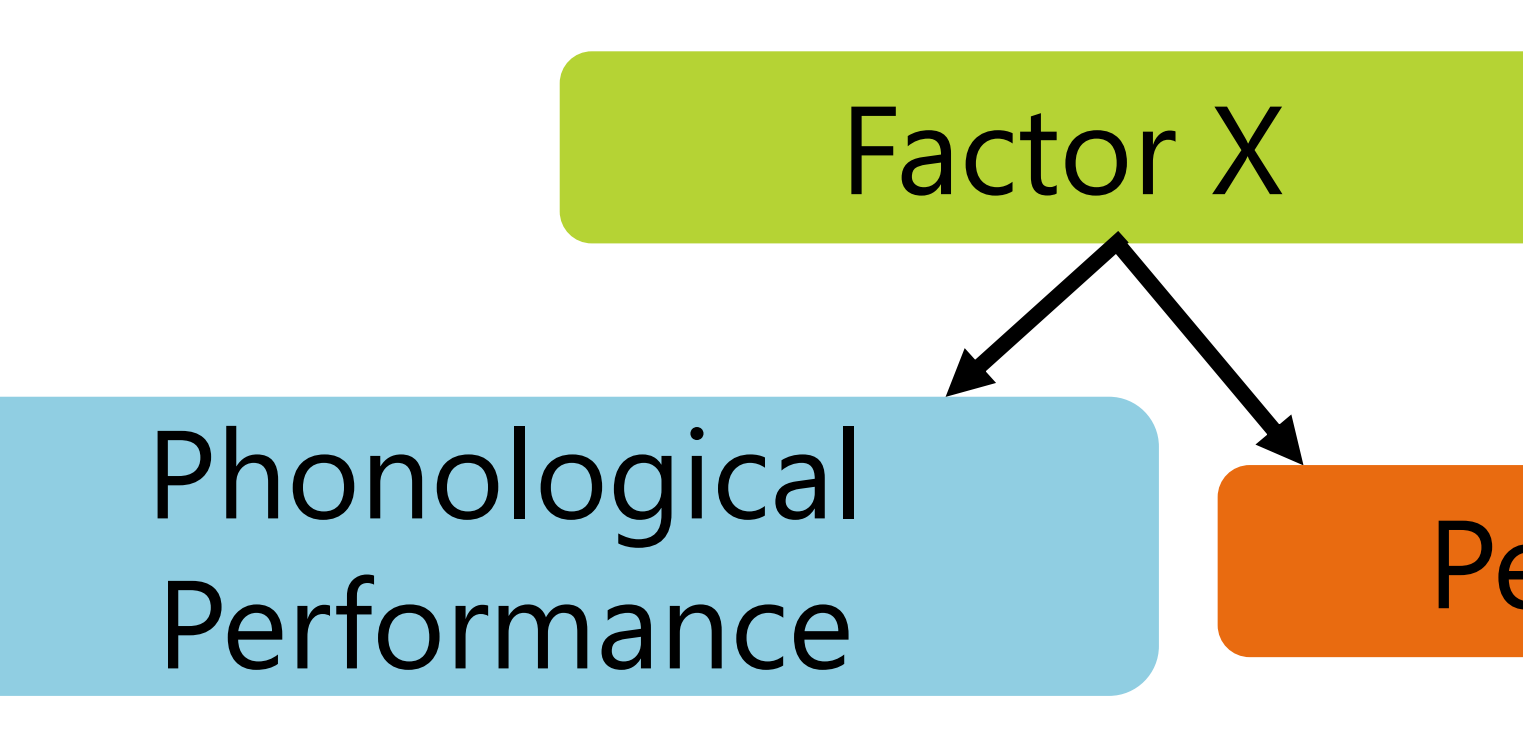

Perception

Q: Are more ambiguous speech sounds harder to maintain in short-term memory?

Contact: harriet.smith@mrc-cbu.cam.ac.uk
We used an automated audio-morphing method (Rogers and Davis, 2014) to create intermediate speech tokens that are a blend of two natural speech signals.

We generated phonetic continua between 10 pairs of letters that differed in one phonetic feature (e.g. ' $B$ ' and ' $D$ ') and 10 pairs of a letter and a word with the same constraints (e.g. ' $B$ ' and 'we').

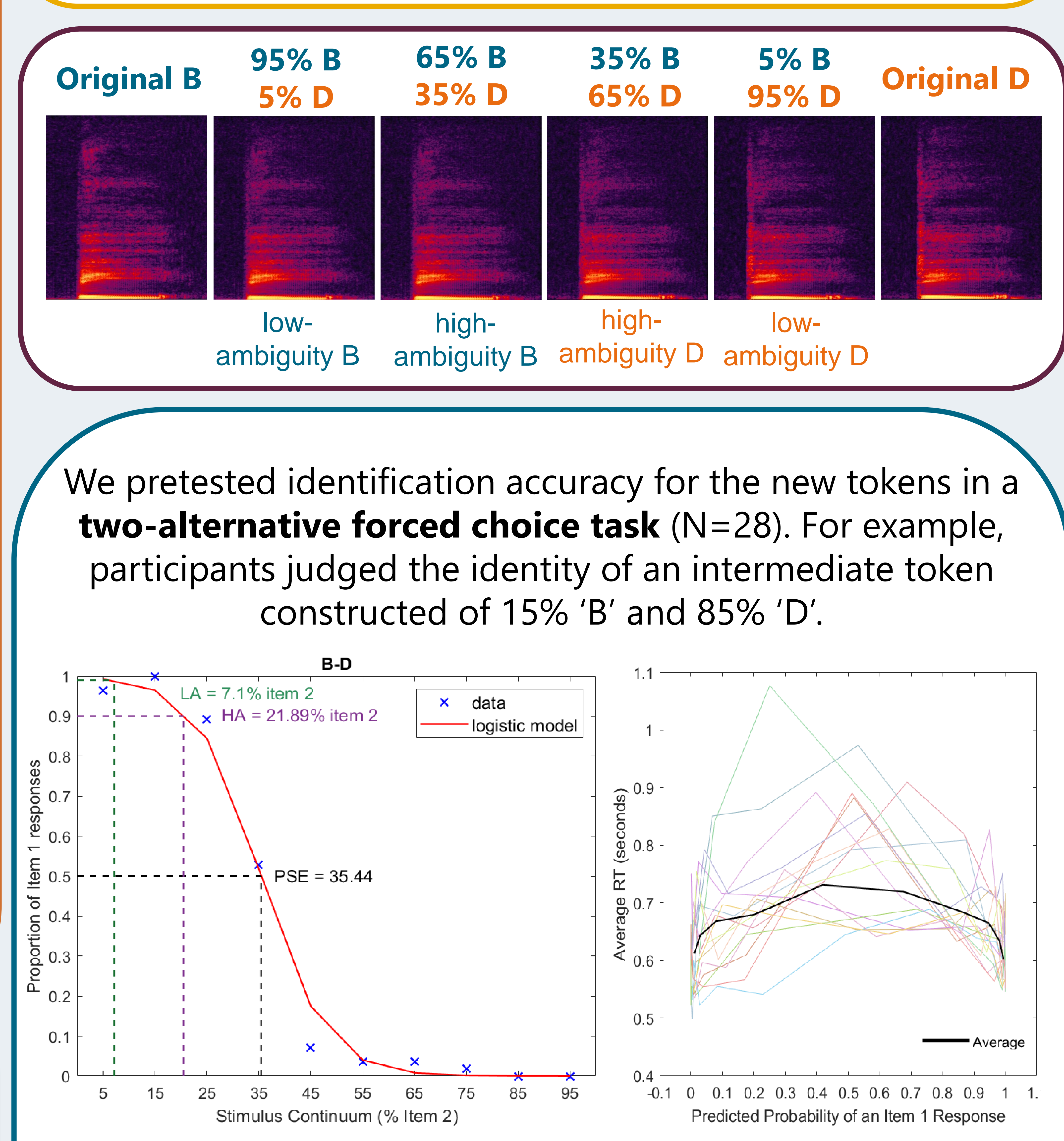

We chose letter stimuli with high and low ambiguity from each continuum, as indexed by predicted probability of a correct response ( 0.99 for low ambiguity items, 0.9 for high ambiguity)

\section{PROCEDURE}

36 British English-speaking adults with no history of language disorder completed an auditory letter-span task.

Participants attempted to recall lists of letters in the order that they heard them. 3 conditions were manipulated:

1. 6- or 8-letter lists

2. Letter stimuli with high or low ambiguity

3. Stimuli from letter-word or letter-letter continua

\section{RESULTS}

Levenshtein distance*: * $a$ string metric for quantifying the difference between two sequences

Recall of low-ambiguity sequences was better than recall of high-ambiguity sequences, but only when ambiguous items were drawn from letter-letter continua (2-way interaction).
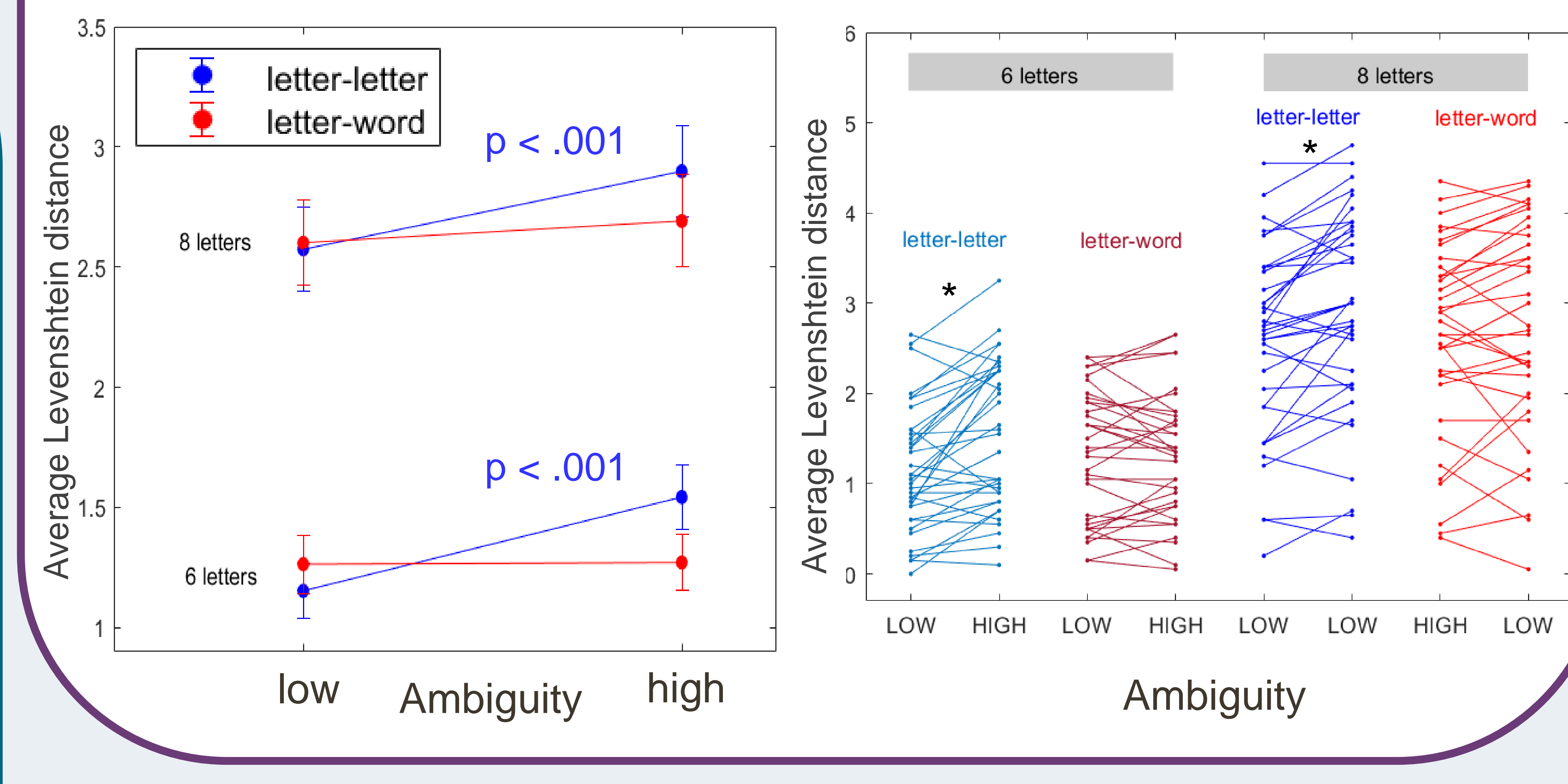

\section{CONCLUSIONS}

Recall of speech is impaired by phonological ambiguity - but only when ambiguity is task-relevant

Highlights potential causal pathway from speech perception to phonological task performance. 